\title{
Slow resolution of inflammation in severe adult dengue patients
}

\author{
Lingzhai Zhao ${ }^{1 \dagger}$, Xiuyan Huang ${ }^{2 \dagger}$, Wenxin Hong ${ }^{1}$, Shuang Qiu', Jian Wang ${ }^{1}$, Lei Yu', Yaoying Zeng ${ }^{2 *}$, \\ Xinghua $\operatorname{Tan}^{1 *}$ and Fuchun Zhang ${ }^{1 *}$
}

\begin{abstract}
Background: The pathogenesis of severe dengue has not been fully elucidated. The inflammatory response plays a critical role in the outcome of dengue disease.

Methods: In this study, we investigated the levels of 17 important inflammation mediators in plasma collected from mild or severe adult dengue patients at different time points to understand the contribution of inflammation to disease severity and to seek experimental evidence to optimize the existing clinical treatment strategies. Patients were simply classified as mild and severe dengue according to the 2009 WHO classification. Plasma was collected on day 3-5, 6-7, 8-10 and 14-17 of illness. Levels of 17 inflammation mediators including TNF-a, IL-1a, IFN- $\gamma$, IL-6, IFN-a, MIF, IL-10, IL-1RA, IL-8, IP-10, MCP-1, RANTES, GRO, eotaxin-1, sICAM-1 and sVCAM-1 were determined by a multiplex Luminex ${ }^{\circledast}$ system. Different trends of inflammation mediators throughout the disease were compared between mild and severe patients.

Results: Inflammation mediators including IL-1a, IFN- $\gamma$, IL-10, IL-8, IP-10, MCP-1 and sVCAM-1 displayed significant differences on day 8-10 of illness between mild and severe dengue patients. Their concentrations were higher in severe patients than mild ones at the same time points. Moreover, those cytokines decreased gradually in mild patients but not in severe patients.

Conclusion: Our results revealed the coexistence of excessive inflammatory response and slow resolution of inflammation in severe adult dengue patients. Hence suppression and/or pro-resolution of inflammation could be a potential therapeutic approach for treatment of severe dengue.
\end{abstract}

Keywords: Severe dengue, Resolution of inflammation, Cytokine, Chemokine, Adhesion molecule

\section{Background}

Dengue, caused by dengue virus (DENV) infection, is considered as the most prevalent mosquito-borne viral disease in humans. An estimated 390 million individuals are infected annually by DENV in tropical and subtropical regions [1]. All four serotypes of DENV (DENV-1 to DENV-4) can cause a spectrum of illness ranging from asymptomatic to life-threatening dengue shock syndrome [2]. Symptomatic disease includes two entities: mild and severe dengue according to the 2009 World

\footnotetext{
* Correspondence: zengyaoying@gmail.com; gz8htxh@126.com; gz8hzfc@ 126.com

${ }^{\dagger}$ Equal contributors

²Department of Immunobiology, Jinan University, Guangzhou, Guangdong 510632, China

'Guangzhou Eighth People's Hospital, Guangzhou Medical University, Guangzhou, Guangdong 510060, China
}

Health Organization (WHO) classification [3]. Mild dengue is a self-limited illness lasting about 7 days. However, severe dengue, characterized by plasma leakage, may be fatal. Although many efforts have been made to elucidate the underlying mechanisms of severe disease, it still remains unclear $[4,5]$.

The inflammatory response against DENV is believed to play an important role in its pathogenesis [6]. The different manifestations between mild and severe dengue patients indicate that inflammatory response may differ substantially. Many studies have demonstrated that levels of inflammation mediators such as TNF- $\alpha$, IFN- $\gamma$, IP-10, IL- 8 are elevated in dengue patients and higher levels in severe cases [7-9]. It has been suggested that inflammation plays a critical role in the outcome of DENV infection. The inflammation is beneficial in 
providing protection against infection and normally terminated once the infection is cleared, while it is detrimental when dysregulated $[10,11]$. Similar to the initiation of inflammation, the resolution of inflammation would be crucial for a successful outcome [12].

Dengue is an expanding public threat to people in South China, where the patients exhibit different symptoms comparative to Southeast Asia. In Southeast Asia, children have greater risk of severe disease while adults show a mild disease. In 2013, China experienced a large dengue outbreak after 1990s, which was especially prevalent in Xishuangbanna region in Yunnan province where an autochthonous dengue outbreak has not been recorded in the last decade and Guangzhou city in Guangdong province where both incidence and case numbers are the highest among Chinese cities [13]. A total of 4432 cases were reported and 86 were severe ones, with an incidence rate of severe dengue of $1.9 \%$ [14]. In this study, we determined levels of 17 important inflammation mediators in plasma collected from mild or severe adult dengue patients at different time points to understand the contribution of inflammation to disease severity and to seek experimental evidence to optimize the existing clinical treatment strategies.

\section{Methods}

\section{Study subjects and samples}

Fifty one dengue patients and 10 healthy volunteers were included in this study from Guangzhou Eighth People's Hospital and Xishuangbanna Dai Autonomous Prefecture People's Hospital between August 20 and November 15 in 2013. All patients were laboratory confirmed to have dengue by dengue virus specific antibody test and real-time PCR assay using serum samples obtained during acute phase of infection. Patients were classified as mild and severe dengue following the 2009 WHO dengue classification. With any of the following conditions, cases were diagnosed as severe disease: (1) plasma leakage leading to shock, respiratory distress, or both; (2) severe bleeding; and (3) severe organ impairment. Plasma of 51 patients was collected on day $3-5$, 6-7, 8-10 and 14-17 of illness. Plasma was collected from 10 age and gender matched healthy volunteers as controls. All samples were stored at $-80{ }^{\circ} \mathrm{C}$. Data on demographic characteristics, clinical features and routine laboratory test findings were also collected.

\section{Diagnosis of primary or secondary infection}

Dengue IgM and IgG capture ELISA kits (Panbio, Brisbane, Queensland, Australia) were used to diagnose primary or secondary dengue infection according to the manufacturer's protocol using serum during acute stage. Dengue-specific IgM positive only was indicative of primary infection. Both $\operatorname{IgM}$ and IgG positive or IgG positive only was diagnosed as secondary infection.

\section{Serotype of DENV assay}

Serotypes of DENV were determined by serotype specific fluorescent PCR diagnostic kits (DAAN Ltd, Guangzhou, Guangdong, China) according to the manufacturer's protocol and performed on an ABI 7500 real-time PCR system (ABI, Foster city, CA).

\section{Measurement of inflammation mediators}

Inflammation mediators were measured using MILLIPLEX ${ }^{\oplus}$ MAP human cytokine/chemokine panel 1 kit and human sepsis panel 1 kit (Merck Millipore, Germany) according to the manufacturer's instructions. Briefly, patients' plasma was mixed with beads coated with capture antibodies to various mediators. Then the mixtures were incubated with biotinylated detection antibodies. Finally, PE-conjugated streptavidin was added, and the fluorescent signals were detected using Luminex ${ }^{\oplus} 200^{\text {TM }}$ System (Life Technologies, Grand Island, NY). Raw data was initially measured as the relative fluorescence intensity and then converted to concentration based on the standard curves generated from the reference concentrations supplied in the kits. 17 inflammation mediators were measured including tumor necrosis factor- $\alpha$ (TNF- $\alpha$ ), interleukin-1 $\alpha$ (IL-1 $\alpha)$, interferon- $\gamma$ (IFN- $\gamma)$, interleukin-6 (IL-6), interferon- $\alpha$ (IFN- $\alpha$ ), macrophage migration inhibitory factor (MIF), interleukin-10 (IL-10), interleuekin-1 receptor antagonists (IL-1RA), interleukin-8 (IL-8), interferon-inducible protein-10 (IP-10), monocyte chemotactic protein-1 (MCP-1), regulated upon activation normal T-cell expressed and secreted (RANTES), growth related oncogene- $\alpha$ (GRO- $\alpha$ ), eotaxin-1, soluble intercellular adhesion molecule-1 (sICAM-1) and soluble vascular cell adhesion molecule-1 (sVCAM-1).

\section{Statistical analysis}

Data are reported as mean \pm standard deviation (SD) for clinical parameters. Statistical analysis was done using GraphPad Prism 5.0 (GraphPad software, San Diego, USA) by $t$ tests or non-parametric tests. Differences of $P<0.05$ were considered statistically significant.

\section{Results}

\section{Characteristics of dengue patients}

Fifty one dengue patients were included in this study. 30 were diagnosed as mild dengue and 21 were severe ones. Detailed demographic, epidemiologic and clinical features of patients are summarized in Tables 1 and 2. All patients were adults. Age was between 18 to 61 years old. Laboratory tests on peripheral blood examination were summarized in Table 2. Results showed that leukopenia especially neutropenia and thrombocytopenia 
Table 1 Demographic and epidemiologic features of mild and severe dengue patients

\begin{tabular}{lll}
\hline Items & Mild $(n=30)$ & Severe $(n=21)$ \\
\hline $\begin{array}{l}\text { Age, median (range) } \\
\text { Gender }\end{array}$ & $35(18-59)$ & $39(18-61)$ \\
$\quad$ Male, no. (\%) & $21(70)$ & $7(33.3)$ \\
$\quad$ Female, no. (\%) & $9(30)$ & $14(66.7)$ \\
Primary or secondary infection & & \\
$\quad$ Primary infection, no. (\%) & $21(70)$ & $11(52.4)$ \\
$\quad$ Secondary infection, no. (\%) & $9(30)$ & $10(47.6)$ \\
DENV serotype & & $10(47.6)$ \\
$\quad$ DENV-1, no. (\%) & $22(73.3)$ & $11(52.4)$ \\
DENV-3, no. (\%) & $8(26.7)$ &
\end{tabular}

occurred in all patients but no increased hematocrit. Albumin was decreased, while alanine aminotransferase (ALT), aspartate aminotransferase (AST), creatine kinase (CK), lactate dehydrogenase (LDH) increased in all patients. Platelet $(\mathrm{PLT})$ was lower $(P=0.048)$ while AST was higher $(P=0.021)$ in severe patients than mild ones.

\section{Levels of inflammatory mediators and dynamic of inflammation resolution at different time points in mild and severe dengue patients}

Levels of 17 inflammation mediators including 6 proinflammatory cytokines, 2 anti-inflammatory cytokines, 7 chemokines and 2 adhesion molecules were determined in mild and severe dengue patients at four time points, on day $3-5,6-7,8-10$ and $14-17$ of disease to observe trends of the mediators over the course of disease. We chose to analyze levels of these mediators on day $6-7$ and $8-10$ of illness in order to understand the difference in inflammation resolution between mild and severe dengue patients.

\section{Pro-inflammatory cytokines}

Six pro-inflammatory cytokines, including TNF- $\alpha, \mathrm{IL}-1 \alpha$, IFN- $\gamma$, IL-6, IFN- $\alpha$ and MIF, were determined. As Fig. 1 shown, concentrations of TNF- $\alpha\{P<0.01$ (Day 3-5), $P<$ 0.01 (Day 6-7), $P<0.01$ (Day 8-10), Fig. 1a\}, IL-1 $\alpha\{P=$ 0.003 (Day 3-5), $P<0.01$ (Day 6-7), $P<0.01$ (Day 810), Fig. 1b $\}$ and IFN- $\gamma\{P=0.047$ (Day $3-5), P=0.074$ (Day 6-7), $P=0.161$ (Day $8-10$ ), Fig. 1c $\}$ were significantly elevated in dengue patients compared to healthy controls at early stage of illness. Then these cytokines exhibited a decreasing trend as patients recovered \{TNF$\alpha: P=0.058$ versus Controls (Day 14-17); IL-1 $\alpha: P=$ 0.094 versus Controls (Day 14-17); IFN- $\gamma: P=0.919$ versus Controls (Day 14-17)\}. Moreover, higher level of TNF- $\alpha(P=0.004)$ on day $6-7$ and higher levels of IL- $1 \alpha$ $(P=0.012)$ and IFN- $\gamma(P=0.001)$ on day $8-10$ of illness
Table 2 Clinical features and laboratory data from mild and severe dengue patients

\begin{tabular}{|c|c|c|}
\hline Items & Mild $(n=30)$ & Severe $(n=21)$ \\
\hline \multicolumn{3}{|l|}{ Clinical features (\%) } \\
\hline Fever & 100 & 100 \\
\hline Rash & 53.3 & 71.4 \\
\hline Myalgia or Arthralgia & 63.3 & 52.4 \\
\hline Hypodynamia & 50 & 100 \\
\hline Vomiting & 16.7 & 28.6 \\
\hline Abdominal pain & 0 & 33.3 \\
\hline Diarrhea & 3.3 & 19 \\
\hline Jaundice & 0 & 14.3 \\
\hline Splenomegalia & 10 & 23.8 \\
\hline Positive tourniquet test & Not detected & 94 \\
\hline Haemorrhagia & 0 & 61.9 \\
\hline Plasma leakage & 0 & 33.3 \\
\hline Hypotension & 0 & 28.6 \\
\hline \multicolumn{3}{|l|}{ Laboratory data (Mean \pm SD) } \\
\hline White Blood Cells count $\left(\times 10^{9} / \mathrm{L}\right)$ & $3.7 \pm 1.5$ & $3.7 \pm 2.1$ \\
\hline Neutrophils count $\left(\times 10^{9} / L\right)$ & $1.9 \pm 1.6$ & $1.4 \pm 0.8$ \\
\hline Lymphocytes count $\left(\times 10^{9} /\llcorner)\right.$ & $1.3 \pm 0.7$ & $1.8 \pm 1.9$ \\
\hline Monocytes count $\left(\times 10^{9} / L\right)$ & $0.4 \pm 0.2$ & $0.4 \pm 0.2$ \\
\hline $\mathrm{CD}^{+} T$ cells count (cells/Ml) & $1026 \pm 552$ & $957 \pm 592$ \\
\hline $\mathrm{CD} 4^{+} T$ cells count $($ cells $/ \mu \mathrm{l})$ & $511 \pm 280$ & $493 \pm 271$ \\
\hline CD8 ${ }^{+} T$ cells count (cells $\left./ \mu l\right)$ & $466 \pm 322$ & $442 \pm 379$ \\
\hline CD4/CD8 ratio & $1.26 \pm 0.80$ & $1.37 \pm 0.68$ \\
\hline Hematocrit (HCT, \%) & $42 \pm 9$ & $39 \pm 6$ \\
\hline Platelet $\left(\times 10^{9} / \mathrm{L}\right)$ & $75 \pm 56$ & $48 \pm 33^{*}$ \\
\hline Total Billirubin $(\mu \mathrm{mol} / \mathrm{L})$ & $12.19 \pm 5.03$ & $13.85 \pm 7.90$ \\
\hline Total Protein (g/L) & $65 \pm 6$ & $62 \pm 6$ \\
\hline Albumin $(g / L)$ & $39 \pm 4$ & $36 \pm 4$ \\
\hline Alanine Transaminase (ALT, U/L) & $60 \pm 71$ & $89 \pm 60$ \\
\hline Aspartate Transaminase (AST, U/L) & $77 \pm 65$ & $126 \pm 71^{*}$ \\
\hline Alkaline Phosphatase (ALP, U/L) & $65 \pm 23$ & $96 \pm 103$ \\
\hline Creatine kinase (CK, U/L) & $203 \pm 140$ & $231 \pm 185$ \\
\hline Lactate Dehydrogenase (LDH, U/L) & $369 \pm 165$ & $490 \pm 271$ \\
\hline Creatinine $(\mu \mathrm{mol} / \mathrm{L})$ & $79 \pm 17$ & $75 \pm 43$ \\
\hline
\end{tabular}

${ }^{*} P<0.05$, severe patients vs mild patients

were observed in severe dengue patients than mild ones. However, IFN- $\alpha(P>0.05$, Fig. $1 \mathrm{~d})$, IL-6 $(P>0.05$, Fig. 1e) and MIF $(P>0.05$, Fig. 1f) did not display significant abnormality at each time point. In addition, no differences of IFN- $\alpha(P>0.05$, Fig. 1d), IL-6 $(P>0.05$, Fig. 1e) and MIF $(P>0.05$, Fig. 1f) were observed on day $6-7$ and on day $8-10$ of illness between mild and severe dengue patients. 


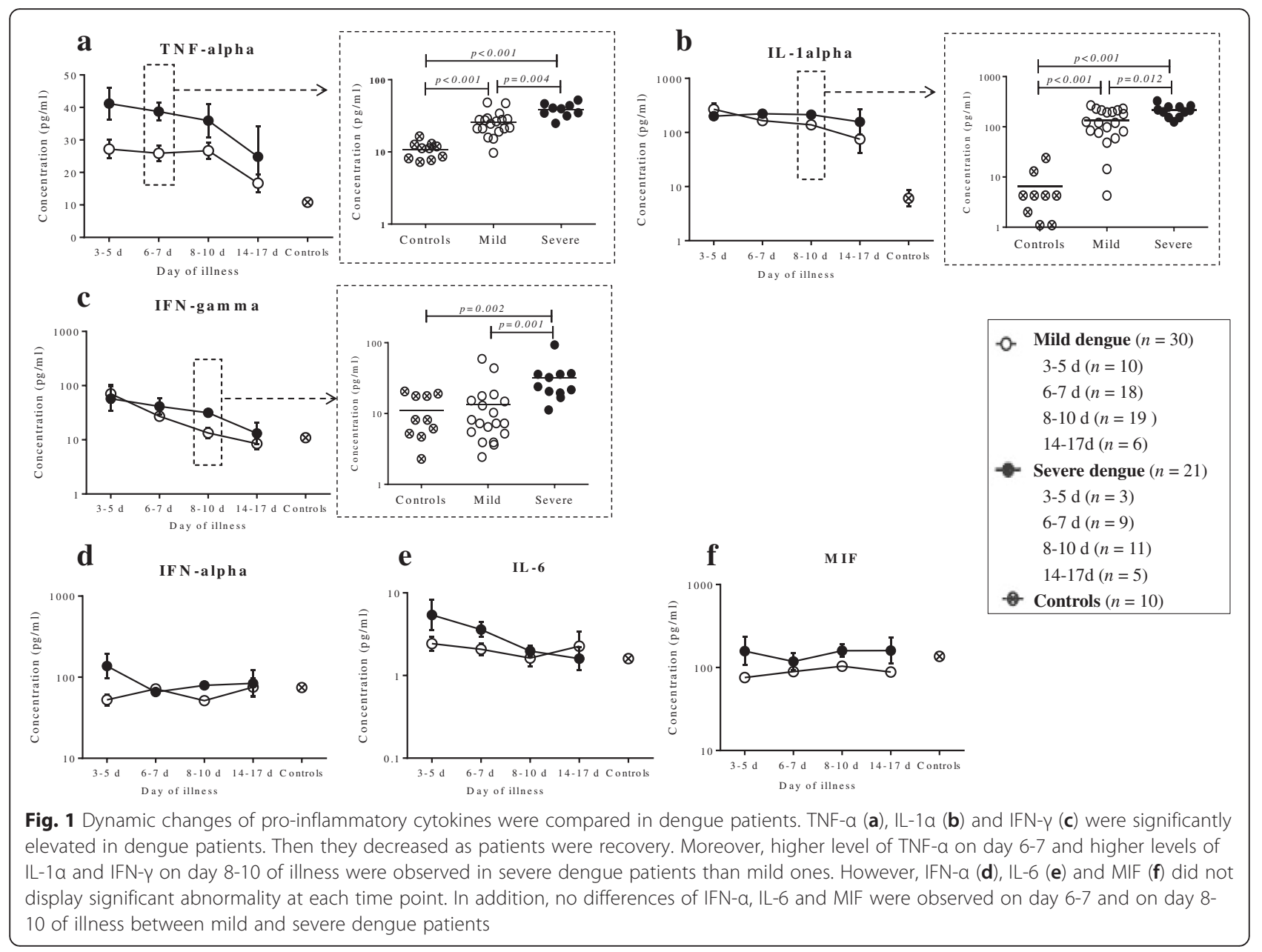

\section{Anti-inflammatory cytokines}

IL-10, as one of the important anti-inflammatory cytokines, was increased at early stage of illness in dengue patients than healthy controls $\{P=0.008$ (Day $3-5), P=$ 0.001 (Day 6-7), $P=0.027$ (Day 8-10), $P=0.098$ (Day 14-17), Fig. 2a\}. However, it showed different decreasing patterns between mild and severe patients (Fig. 2a). Mild dengue patients displayed decreased trends after day 67 of illness (Fig. 2a). In contrast, increased trends were observed in severe dengue patients (Fig. 2a). The concentration of IL-10 was significantly higher in severe than mild patients on day 8-10 of illness $(P=0.003$,

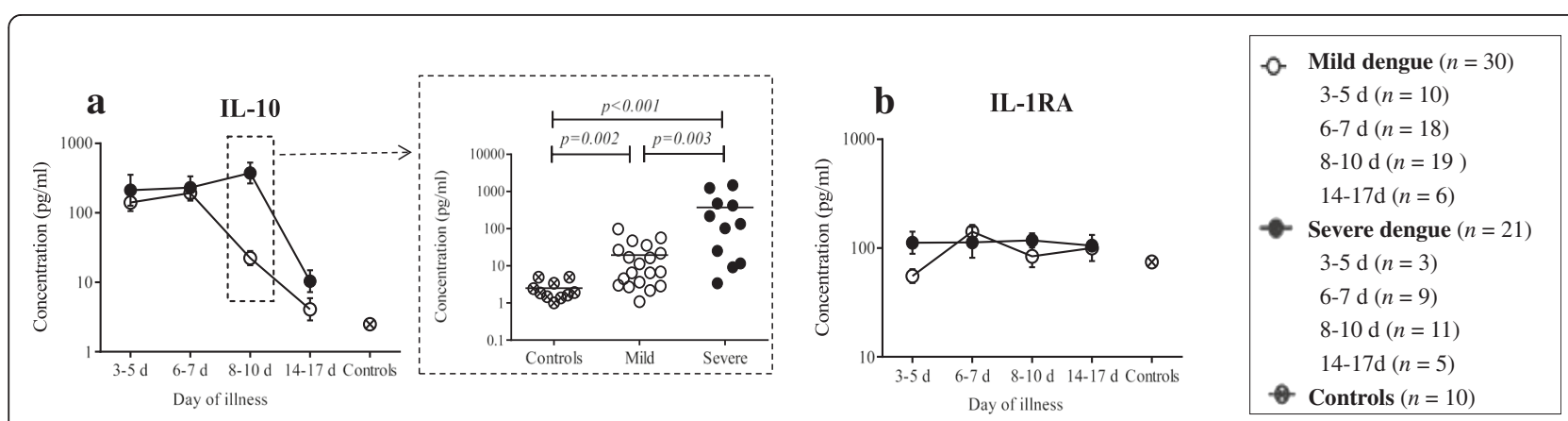

Fig. 2 Different trends of anti-inflammatory cytokines were observed between mild and severe dengue patients. IL-10 was increased at early stage of illness in dengue patients. However, it showed different decreasing patterns between mild and severe patients (a). No abnormality was observed with regard to IL-1RA at each time points between dengue patients and healthy controls (b) 
Fig. 2a). No significant difference was observed between dengue patients and healthy controls with respect to plasmatic IL-1RA concentration $(P>0.05$, Fig. $2 b)$.

\section{Chemokines}

Seven chemokines, including IL-8, IP-10, MCP-1, RANTES, GRO- $\alpha$, eotaxin- 1 and MDC, were detected. IL-8 was higher in severe patients than mild patients and showed decreasing trends as patients recovered $\{(P=0.008$ (Day 6-7), $P=0.007$ (Day $8-10$ ), Fig. 3c $\}$. IP-10 $\{P<0.01$ (Day 3-5), $P<0.01$ (Day 6-7) $P<0.01$ (Day $8-10$ ), $P=0.037$ (Day 14-17), Fig. 3a $\}$ and MCP-1
$\{P<0.01$ (Day 3-5), $P<0.01$ (Day 6-7), $P=0.040$ (Day $8-10$ ), $P=0.500$ (Day 14-17), Fig. 3b $\}$ were higher in dengue patients compared with healthy controls. They displayed similar decreasing trends throughout the disease progress. Significant differences of IP-10 $(P=$ 0.002 , Fig. 3a) and MCP-1 $(P=0.007$, Fig. $3 \mathrm{~b})$ were noted between mild and severe dengue patients on day $8-10$, with continually decreasing trends in mild patients but not in severe patients. Level of RANTES was lower in severe dengue patients compared to mild patients and healthy controls on day $6-7(P=0.002$ versus healthy controls, $P=0.024$ versus mild patients) and 8 -

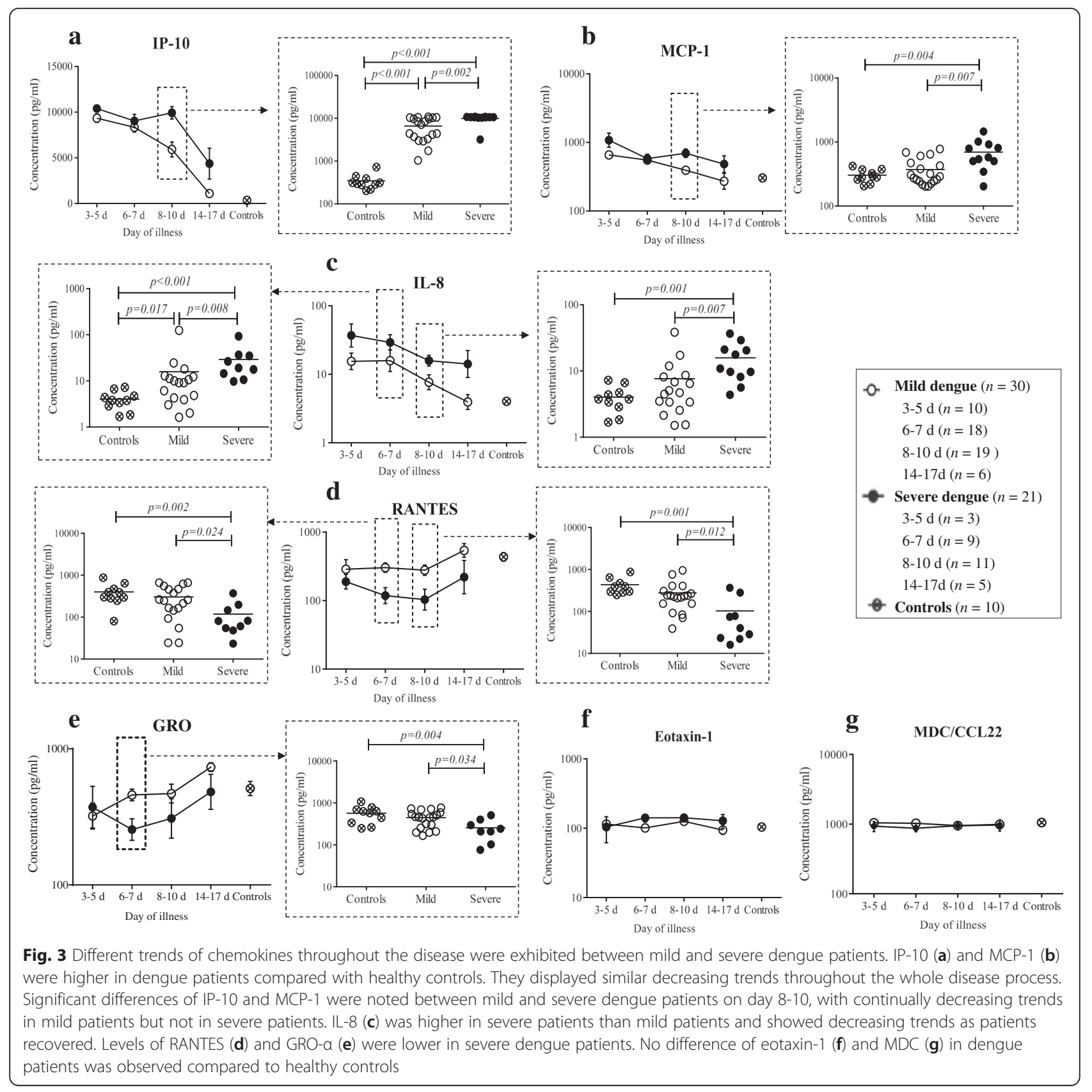


$10(P=0.001$ versus healthy controls, $P=0.012$ versus mild patients) of illness (Fig. 3d). GRO- $\alpha$ was similar to RANTES but a significant difference was noted only on day 6-7 of illness between mild and severe dengue patients $(P=0.034$, Fig. 3e). No difference was observed between dengue patients and healthy controls in eotaxin-1 $(P>0.05$, Fig. 3f $)$ and MDC $(P>0.05$, Fig. 3g).

\section{Adhesion molecules}

With regard to adhesion molecules, sVCAM- $1\{P<0.01$ (Day 3-5), $P<0.01$ (Day 6-7), $P<0.01$ (Day $8-10$ ), $P=$ 0.036 (Day 14-17), Fig. 4a\} were significantly higher and sICAM-1 $(P>0.05$, Fig. 4b) were normal in dengue patients compared to healthy controls. Different trends of sVCAM-1 were observed between mild and severe patients as shown in Fig. 4a. Level of sVCAM-1 was displayed decreasing trend after day 6-7 in mild dengue patients while after day 8-10 in severe ones (Fig. 4a).

\section{Discussion}

As we know, inflammation is an innate immune response to infection and supposed to be protective. However, nonresolving inflammation is a major trigger of disease and turns destructive [15]. Hence multiple mechanisms are evolved to resolve inflammation. Effector cells normally undergo apoptosis or differentiate to other phenotypes. Cells like macrophages could polarize from M1 towards M2 [16]. M1 macrophages play key roles in triggering inflammation via secreting pro-inflammatory factors, while M2 macrophages involve in immunosuppression and tissue repair by releasing series of anti-inflammatory mediators [17]. Discordance of those resolution mechanisms might result in excessive inflammation and detrimental outcomes. In this study, the expression profile of several important inflammation mediators, including TNF- $\alpha$, IL-1 $\alpha$, IL-1, IFN- $\gamma$ and IL-10, was investigated in dengue patients at different time points. Our results showed that there are significant differences between mild and severe patients. Those results provided solid evidence that inflammation played a pivotal role in determining the severity of dengue disease.

Once infected by DENV, the hosts immediately triggered an immune response to eliminate the virus. Similar to DENV, infections by other flaviviruses such as West Nile Virus (WNV) and Japanese encephalitis virus (JEV) also induce robust proinflammatory cytokine responses to cause immunopathology [18]. An appropriate inflammatory response is protective and essential for resistance to infection. Decreased or absent inflammatory response may lead to propagation of virus and progression of disease. However, excessive inflammatory response is believed to play a direct role in the pathogenesis of severe dengue disease $[19,20]$. Studies have been reported that severe dengue patients display uncontrolled immune cell activation and increased inflammation mediators production. Elevated mediators change the activation status and function of the vascular endothelium and eventually lead to transient increase in vascular permeability $[20,21]$. We also found high levels of inflammation mediators in severe patients in this study.

TNF- $\alpha$, as a classic pro-inflammatory cytokine, stimulates the acute phase reaction. In this study elevated TNF- $\alpha$ was observed in all dengue patients but higher in severe cases. The important role of TNF- $\alpha$ in DENV infection has been reported in many previous studies $[8,22]$. Although its inhibitory effect on DENV replication is benefit, TNF- $\alpha$ may also account for activation of vascular endothelial cell and increase vascular permeability. TNF- $\alpha$ has been associated with hemorrhagic manifestations of dengue [23]. Anti-TNF- $\alpha$ antibodies treatment of DENV-infected mouse has shown effects on preventing severe symptoms and reducing mortality [24]. However, inconsistency or no differences of TNF$\alpha$ between different disease severity, even compared with healthy controls have also been reported in some studies [7]. This discrepancy could be due to TNF- $\alpha$ genetic polymorphisms or differences of study population. IL- $1 \alpha$, produced mainly by activated macrophages,

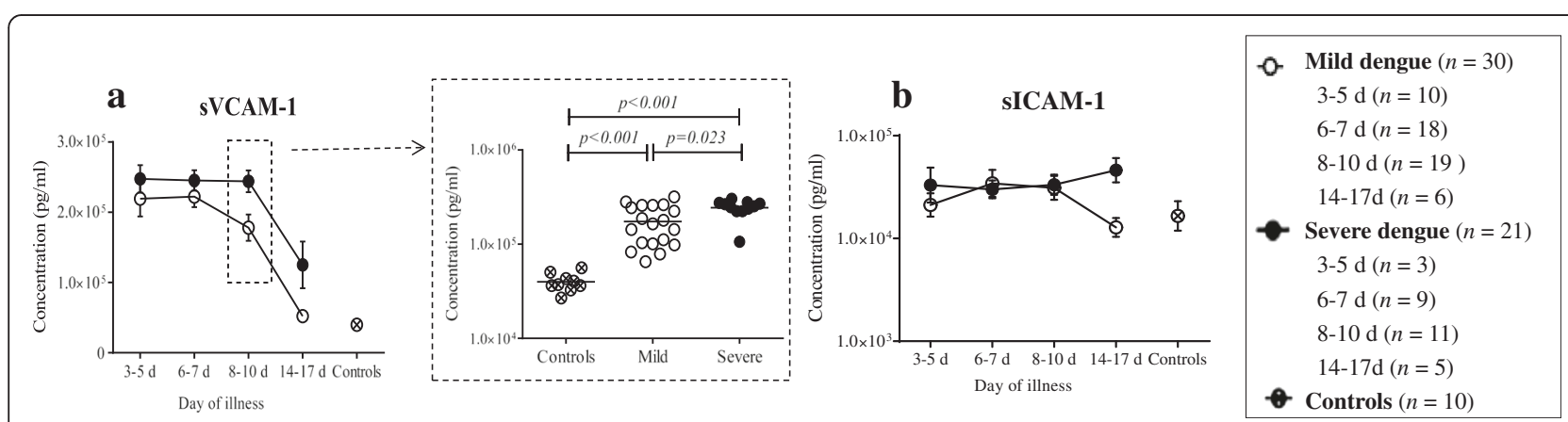

Fig. 4 Different trends of adhesion molecules were compared between mild and severe dengue patients. SVCAM-1 (a) were higher and sICAM-1 (b) were normal in dengue patients compared to healthy controls. Different trends of sVCAM-1 were observed between mild and severe patients 
is a member of IL- 1 family like IL- $1 \beta$. Increased IL- $1 \beta$ in severe patients has been reported when compared to mild cases [8]. However, Level of IL-1 $\alpha$ in dengue patients has not been reported. In this study, increased IL- $1 \alpha$ during the course of illness was observed. There were significant differences between mild and severe cases on day 8-10 of illness. IFN- $\gamma$ has been reported to be associated with severity in many previous studies $[25,26]$. IFN- $\gamma$ displayed similar trend as IL- $1 \alpha$ in this study. IL-10, as a central anti-inflammatory cytokine, suppresses the expression of pro-inflammatory cytokines, chemokines and adhesion molecules. Some study found that level of IL-10 was higher during febrile phase and correlate with the degree of plasma leakage $[27,28]$. But other study reported that IL-10 peaked during defervescence as the body attempted to control the acute systemic inflammatory response [26]. In this study we observed that IL-10 peaked on day 6-7 in mild patients, while in severe cases on day $8-10$ of illness. This discrepancy of IL-10 provided evidence of differences of inflammatory response between mild and severe patients. Chemokines also displayed differences between mild and severe dengue patients in this study. IL-8 is a chemokine produced mainly by macrophages. Similar to TNF- $\alpha$, IL- 8 is an innate immune mediator and mediates acute phase reaction. The effects on pathogenesis of severe dengue has been reported [26]. The trend of IL- 8 in dengue patients was similar to TNF- $\alpha$ in this study. IP-10 is secreted by several cell types such as monocytes in response to IFN- $\gamma$ and has important role in disease initiation and progression. Abnormal levels of IP-10 have been associated with inflammatory diseases [29]. In this study we observed significantly higher levels of IP-10 in dengue patients. The levels declined steadily in mild cases throughout the disease. However, the levels rose again in severe cases on day 8-10 of illness. This suggested that recurrent inflammatory response happened in severe cases. It can explain the trend of IL-10 at the same time point. Peaks of pro-inflammatory cytokines and peaks of antiinflammatory cytokines coexist. MCP-1 is a potent monocytes chemotractant and has been reported that increased levels correlated with severe dengue symptoms [30]. The results in this study also proved that inflammation in severe dengue patients resolved differently from mild cases. However, lower levels of GRO- $\alpha$ and RANTES were observed in severe patients on day 6-7 than mild cases in this study. A recent study reported that RANTES levels were decreased in dengue patients and correlated strongly with platelets count and disease severity, similar to our results [9]. Lower levels of RANTES in dengue patients could be due to thrombocytopenia during defervescence particularly in severe cases. That was because GRO- $\alpha$ and RANTES were released by activated platelet [31]. Another study also proved that platelets from DENV infected patients secreted lower levels of RANTES in vitro than did platelets from healthy individuals [32]. The mechanism of decreased GRO- $\alpha$ may be the same as RANTES.

sVCAM-1 is released from the endothelial cell surface into the circulation upon endothelial activation and now proved to be a marker for endothelial injury under inflammatory processes [33]. Elevated levels of sVCAM-1 have been reported in dengue patients and associate with severity of disease [34]. In this study higher levels in severe dengue patients were observed than mild patients on day 8-10 of illness. Increased sVCAM-1 levels in all dengue patients indicated that abnormal function of vascular endothelial cells occurred after onset of illness as reported in another study. Evidence from serial ultrasound studies also indicated that plasma leakage actually started at initial stage of disease in all patients [35]. It is possible that most of patients experience some degree of plasma leakage and only a minority gets worse.

There is no specific antiviral treatment and vaccine currently available for DENV infection. Treatment is largely supportive to alleviate of symptoms and prevent shock by fluid resuscitation. Further investigations of the mechanisms of severe disease may provide insight into novel clinical management strategies. A number of studies have shown excessive inflammation mediators in severe dengue patients [36]. Inflammation mediators during DENV infection, like a double-edged sword, though anti-virus, may also account for increased vascular permeability that characterizes severe dengue $[37,38]$. Defervescence, on around day $6-7$ of dengue disease, is a watershed for disease progression that patients can either recover rapidly or progress to a severe life-threatening stage. As we observed in this study, most significant differences were noted on day 8-10 of illness between mild and severe dengue patients. That is, inflammation was very rapidly resolved in mild patients but not in severe cases. So suppression or pro-resolution of inflammation could be a potential therapeutic approach for treatment of severe dengue.

\section{Conclusion}

Our results revealed the coexistence of excessive inflammatory response and slow resolution of inflammation in severe adult dengue patients. Thus, excessive inflammatory response or failed termination of inflammation usually contributes to the pathogenesis of severe dengue disease. Therefore, it is possible to introduce drugs with anti-inflammatory or pro-resolving effects to current therapeutic regimen, which might prevent severe disease development and decrease dengue mortality. Our study provides substantial evidence to support such hypothesis. 


\section{Abbreviations}

ALT, Alanine aminotransferase; AST, Aspartate aminotransferase; CK, Creatine kinase; DENV, Dengue Virus; GRO, Growth related oncogene; IFN-a, Interferon-a; IFN- $\gamma$, Interferon- $\gamma ;$ IL-10, Interleukin-10; IL-1RA, Interleuekin-1 receptor antagonists; IL-1a, Intereukin-1a; IL-6, Interleukin-6; IL-8, Interleukin8; IP-10, Interferon-inducible protein-10; JEV, Japanese encephalitis virus; LDH, Lactate dehydrogenase; MCP-1, Monocyte chemotactic protein-1; MIF, Macrophage migration inhibitory factor; PLT, Platelet; RANTES, Regulated upon activation normal T-cell expressed and secreted; SD, Standard deviation; sICAM-1, soluble intercellular adhesion molecule-1; sVCAM-1, soluble vascular cell adhesion molecule-1; TNF-a, Tumor necrosis factor-a; $\mathrm{WHO}$, World health organization; WNV, West nile virus

\section{Acknowledgments}

We thank Allen KL Cheung from The University of Hong Kong for manuscript editing

\section{Funding}

This work was supported by National "12th Five Year Plan" Mage Scientific and Technology Program Grant (2012ZX10004301-003) and grant from the National Natural Science Foundation of China (31200667)

\section{Availability of data and materials}

Raw data can be made available to interested researchers on request to the corresponding authors.

\section{Authors' contributions}

FCZ, XHT and YYZ conceived and designed the study. LZZ and XYH performed the experiments and wrote the paper. SQ collected samples and laboratory data. JW and FCZ contributed to the classification of patients. WXH and LY contributed to analyze data and carefully read the draft. All authors read and approved the final manuscript.

\section{Competing interests}

The authors declare that they have no competing interests.

\section{Consent for publication}

Not applicable.

\section{Ethics approval and consent to participate}

Ethical approval for the study was obtained from the institutional review board of Guangzhou Eighth People's Hospital. Written informed consent was obtained from all research participants.

\section{Received: 28 October 2015 Accepted: 26 May 2016}

Published online: 14 June 2016

\section{References}

1. Bhatt S, Gething PW, Brady OJ, Messina JP, Farlow AW, Moyes CL, Drake JM, Brownstein JS, Hoen AG, Sankoh O, et al. The global distribution and burden of dengue. Nature. 2013;496(7446):504-7

2. Simmons CP, Farrar JJ, Nguyen W, Wills B. Dengue. N Engl J Med. 2012; 366(15):1423-32

3. Dengue: Guidelines for Diagnosis, Treatment, Prevention and Control: New Edition. edn. Geneva; 2009.

4. Wilder-Smith A. Dengue vaccines: dawning at last? Lancet. 2014;384(9951) 1327-9.

5. St John AL, Abraham SN, Gubler DJ. Barriers to preclinical investigations of anti-dengue immunity and dengue pathogenesis. Nat Rev Microbiol. 2013; 11(6):420-6.

6. Costa W, Fagundes $C T$, Souza DG, Teixeira MM. Inflammatory and innate immune responses in dengue infection: protection versus disease induction. Am J Pathol. 2013;182(6):1950-61.

7. Rathakrishnan A, Wang SM, Hu Y, Khan AM, Ponnampalavanar S, Lum LC, Manikam R, Sekaran SD. Cytokine expression profile of dengue patients at different phases of illness. PLoS One. 2012;7(12):e52215.

8. Arias J, Valero N, Mosquera J, Montiel M, Reyes E, Larreal Y, Alvarez-Mon M. Increased expression of cytokines, soluble cytokine receptors, soluble apoptosis ligand and apoptosis in dengue. Virology. 2014;452-453:42-51.

9. van de Weg CA, Pannuti CS, de Araujo ES, van den Ham HJ, Andeweg AC, Boas LS, Felix AC, Carvalho Kl, de Matos AM, Levi JE, et al. Microbial translocation is associated with extensive immune activation in dengue virus infected patients with severe disease. PLoS Negl Trop Dis. 2013;7(5): e2236.

10. Medzhitov R. Origin and physiological roles of inflammation. Nature. 2008; 454(7203):428-35.

11. Serhan CN, Brain SD, Buckley CD, Gilroy DW, Haslett C, O’Neill LA, Perretti M, Rossi AG, Wallace JL. Resolution of inflammation: state of the art, definitions and terms. FASEB J. 2007;21(2):325-32.

12. Buckley CD, Gilroy DW, Serhan CN, Stockinger B, Tak PP. The resolution of inflammation. Nat Rev Immunol. 2013;13(1):59-66.

13. Zhang FC, Zhao H, Li LH, Jiang T, Hong WX, Wang J, Zhao LZ, Yang HQ, Ma DH, Bai CH, et al. Severe dengue outbreak in Yunnan, China, 2013. Int J Infect Dis. 2014:27:4-6.

14. Prevention CCfDCa: Reportable infectious disease statistics, Beijing. In.; 2013.

15. Medzhitov R. Inflammation 2010: new adventures of an old flame. Cell. 2010;140(6):771-6.

16. Ortega-Gomez A, Perretti M, Soehnlein O. Resolution of inflammation: an integrated view. EMBO Mol Med. 2013;5(5):661-74

17. Liu YC, Zou XB, Chai YF, Yao YM. Macrophage polarization in inflammatory diseases. Int J Biol Sci. 2014;10(5):520-9.

18. Winter PM, Dung NM, Loan HT, Kneen R, Wills B, le Thu T, House D, White NJ, Farrar JJ, Hart CA, et al. Proinflammatory cytokines and chemokines in humans with Japanese encephalitis. J Infect Dis. 2004;190(9):1618-26.

19. Guabiraba R, Ryffel B. Dengue virus infection: current concepts in immune mechanisms and lessons from murine models. Immunology. 2014;141(2): 143-56.

20. Rothman AL. Immunity to dengue virus: a tale of original antigenic sin and tropical cytokine storms. Nat Rev Immunol. 2011;11(8):532-43.

21. Tolfvenstam T, Lindblom A, Schreiber MJ, Ling L, Chow A, Ooi EE, Hibberd ML. Characterization of early host responses in adults with dengue disease. BMC Infect Dis. 2011;11:209.

22. Levy A, Valero N, Espina LM, Anez G, Arias J, Mosquera J. Increment of interleukin 6, tumour necrosis factor alpha, nitric oxide, C-reactive protein and apoptosis in dengue. Trans R Soc Trop Med Hyg. 2010; 104(1):16-23

23. Cardier JE, Marino E, Romano E, Taylor P, Liprandi F, Bosch N, Rothman AL. Proinflammatory factors present in sera from patients with acute dengue infection induce activation and apoptosis of human microvascular endothelial cells: possible role of TNF-alpha in endothelial cell damage in dengue. Cytokine+. 2005:30(6):359-65.

24. Shresta S, Sharar KL, Prigozhin DM, Beatty PR, Harris E. Murine model for dengue virus-induced lethal disease with increased vascular permeability. J Virol. 2006;80(20):10208-17.

25. Pal T, Dutta SK, Mandal S, Saha B, Tripathi A. Differential clinical symptoms among acute phase Indian patients revealed significant association with dengue viral load and serum IFN-gamma level. J Clin Virol. 2014;61(3):365-70.

26. Pandey N, Jain A, Garg RK, Kumar R, Agrawal OP, Lakshmana Rao PV. Serum levels of IL-8, IFNgamma, IL-10, and TGF beta and their gene expression levels in severe and non-severe cases of dengue virus infection. Arch Virol. 2015;160(6):1463-75.

27. Al-Harthi L, Siegel J, Spritzler J, Pottage J, Agnoli M, Landay A. Maximum suppression of HIV replication leads to the restoration of HIV-specific responses in early HIV disease. AIDS. 2000;14(7):761-70.

28. Malavige GN, Gomes L, Alles L, Chang T, Salimi M, Fernando S, Nanayakkara KD, Jayaratne S, Ogg GS. Serum IL-10 as a marker of severe dengue infection. BMC Infect Dis. 2013;13(1):341.

29. Liu M, Guo S, Hibbert JM, Jain V, Singh N, Wilson NO, Stiles JK. CXCL10/IP-10 in infectious diseases pathogenesis and potential therapeutic implications. Cytokine Growth Factor Rev. 2011;22(3):121-30.

30. Lee YR, Liu MT, Lei HY, Liu CC, Wu JM, Tung YC, Lin YS, Yeh TM, Chen SH, Liu HS. MCP-1, a highly expressed chemokine in dengue haemorrhagic fever/dengue shock syndrome patients, may cause permeability change, possibly through reduced tight junctions of vascular endothelium cells. J Gen Virol. 2006:87(Pt 12):3623-30.

31. Gear AR, Camerini D. Platelet chemokines and chemokine receptors: linking hemostasis, inflammation, and host defense. Microcirculation. 2003;10(3-4): $335-50$

32. Hottz ED, Medeiros-de-Moraes IM, Vieira-de-Abreu A, de Assis EF, Vals-deSouza R, Castro-Faria-Neto HC, Weyrich AS, Zimmerman GA, Bozza FA, Bozza PT. Platelet activation and apoptosis modulate monocyte inflammatory responses in dengue. J Immunol. 2014;193(4):1864-72. 
33. Page AV, Liles WC. Biomarkers of endothelial activation/dysfunction in infectious diseases. Virulence. 2013;4(6):507-16.

34. Murgue B, Cassar O, Deparis X. Plasma concentrations of sVCAM-1 and severity of dengue infections. J Med Virol. 2001;65(1):97-104.

35. Michels M, Sumardi U, de Mast Q, Jusuf H, Puspita M, Dewi IM, Sinarta S, Alisjahbana B, van der Ven AJ. The predictive diagnostic value of serial daily bedside ultrasonography for severe dengue in Indonesian adults. PLoS Negl Trop Dis. 2013;7(6):e2277.

36. Martina BE, Koraka P, Osterhaus AD. Dengue virus pathogenesis: an integrated view. Clin Microbiol Rev. 2009;22(4):564-81.

37. Butthep P, Chunhakan S, Yoksan S, Tangnararatchakit K, Chuansumrit A. Alteration of cytokines and chemokines during febrile episodes associated with endothelial cell damage and plasma leakage in dengue hemorrhagic fever. Pediatr Infect Dis J. 2012;31(12):e232-8.

38. Tisoncik JR, Korth MJ, Simmons CP, Farrar J, Martin TR, Katze MG. Into the eye of the cytokine storm. Microbiol Mol Biol Rev. 2012;76(1):16-32.

Submit your next manuscript to BioMed Central and we will help you at every step:

- We accept pre-submission inquiries

- Our selector tool helps you to find the most relevant journal

- We provide round the clock customer support

- Convenient online submission

- Thorough peer review

- Inclusion in PubMed and all major indexing services

- Maximum visibility for your research

Submit your manuscript at www.biomedcentral.com/submit
Biomed Central 